\title{
Internes Coaching in der Gruppe
}

\section{Eine Studie zu Wirkungsbedingungen, Auswirkungen und Effekten auf die Unternehmenskultur}

\author{
Günther Mohr
}

Zusammenfassung: Für ein internes Coaching-System in einem Unternehmen wurde eine Studie zu den Wirkbedingungen und den Auswirkungen von Coaching durchgeführt. Zunächst wird mithilfe der QCA (Qualitative Comparative Analysis) nach Kagin der Zusammenhang zwischen unabhängigen Variablen der Teilnahme an der Gruppe und der abhängigen Variable der „Übertragung zusätzlicher Verantwortung" durch die Firma betrachtet. Die zweite Stufe besteht in einer inhaltsanalytischen Betrachtung einer Befragung der Teilnehmer zu Wirkbedingungen und Effekten der Coaching-Gruppen. Die dritte Stufe enthält den Vergleich mit einer strukturierten Befragung zu genauen Kriterien der Auswirkung der Coaching-Gruppen, in wieweit innerhalb eines Jahres die wichtigste professionelle Fragestellung des jeweiligen Teilnehmers vorangebracht werden konnte. Das Ergebnis ergab eine Fülle von Anregungen und zeigt einige Kernfaktoren eines solchen Coaching-Systems.

Schlüsselwörter: Internes coaching · Gruppen-Coaching $\cdot$ Wirkungsforschung

\section{Inhouse coaching in groups - A study to conditions, impacts and effects to the company culture}

\begin{abstract}
The article presents a study of an Inhouse coaching system in a regional service company. After a description of the particular coaching system in groups the conditions, impacts and effects of the coaching is looked at by three steps: First a QCA (Qualitative Comparative Analysis) following Kagin focusses the connection between factors like behavior of participation and the by the company given responsibility. Secondly a content analysis of the view of the participants reveals conditions for a successful coaching. Thirdly an additional survey of the participants in a half structured form regarding specific results like dealing with main challenge of the year is added. The results give insight in the designing of good coaching processes as a contribution and a piece of organizational culture in a company.
\end{abstract}

Keywords: Inhouse coaching · Group coaching $\cdot$ Effectivity research

Online publiziert: 17.07 .2012

(C) Die Autor(en) 2012. Dieser Artikel ist auf Springerlink.com mit Open Access verfügbar.

Dipl.-Psych. G. Mohr $(\bowtie)$

Klarastr. 7, 65719 Hofheim, Deutschland

E-Mail: info@mohr-coaching.de 


\section{Das Coaching-System}

Die Form des Coachings - Gruppen-Coaching: Der DBVC (Deutscher Bundesverband Coaching) beschreibt unter den Coaching-Settings Gruppen-Coaching als „Coaching-Variante, bei der mehrere Personen, die keine gegenseitigen Abhängigkeiten haben, in einer Gruppe gecoacht werden. Die Anliegen sind dabei thematisch so ausgerichtet, dass sinnvolles und effektives Lernen der Gruppenteilnehmer voneinander und miteinander gewährleistet ist" (DBVC 2010, S.29). Das im hier vorgestellten Projekt praktizierte Coaching-System kann man in diesem Sinne als Gruppen-Coaching bezeichnen. Doch zunächst einige Informationen zum systemischen Kontext, ohne den eine Einordnung des Coaching-Systems nicht möglich ist.

Das Unternehmen: Das Unternehmen gehört zur Kategorie „regionale Dienstleister“ für Privat- und Firmenkunden mit stationärem Vertrieb. Es war nach traditionsreicher Geschichte in den letzten zehn Jahren in einige Turbulenzen geraten und vor fünf Jahren auch unter Einflussnahme der Politik von einem größeren Konzern übernommen worden. Das Teilunternehmen wurde aber aufgrund von Produktverschiedenheit nicht integriert, sondern konnte im Rahmen der Erreichung finanzieller Ziele als eigenständige Einheit weiterarbeiten. Das Unternehmen selbst hatte zum Zeitpunkt der Studie 1600 Mitarbeiter/ innen und ungefähr 250 Führungskräfte auf vier Hierarchieebenen.

Rahmenbedingungen des Marktes: Der Markt des Unternehmens war durch die Finanz- und Wirtschaftskrise sehr betroffen. Die Kunden waren mit den Produkten des Unternehmens vorsichtig geworden. Stabilisierend wirkte, dass viele Kunden auf Basisdienstleistungen in diesem Markt angewiesen waren und noch eine relative treue Kundschaft darstellten, obwohl die Abwanderung in den Onlinebereich langsam, aber stetig zunahm. Die durch die Grundversorgung der treuen Kunden fließenden Bestandserträge bildeten die Basis der Erträge. Darüber hinaus wurde ständig versucht, durch ausgefeiltere Produkte zusätzliches Geschäft zu generieren.

Systemische Rahmenbedingungen des internen Coachings - „Wir leisten uns Sie“: Im Unternehmen gab es eine lange Tradition der innovativen Bildungsarbeit. Diese Tradition reichte - um eine Vorstellung davon zu vermitteln - 150 Jahre zurück. Man hatte sich auch immer für die Nachwuchsförderung engagiert. Dies beinhaltete v.a. die Entwicklung der eigenen Mitarbeiter. Es wurden nur sehr wenige Arbeitskräfte von außen, etwa mit Universitätsabschluss, eingestellt. Menschen wurden mittels des internen Bildungssystems für das Unternehmen sozialisiert. Erfahrungsgemäß förderte dies auch eine lange Betriebszugehörigkeit. Das Referat Coaching und Praxisberatung wurde von mir selbst im Unternehmen angeregt und im Jahre 1999 eingerichtet. Dies war ein Novum, aber v. a. durch meine vorherige Präsenz in anderen Abteilungen des Unternehmens möglich. Man kannte mich aus Führungstrainings und anderer Personalentwicklungsarbeit, hatte Vertrauen, das war wichtig. Der Vorstandssprecher machte dazu einmal - einschränkend, dass er selbst von der Materie wenig Ahnung hätte, aber diese offensichtlich heute wichtig sei - die Bemerkung: „Wir leisten uns Sie“. 


\section{Die Prinzipien der internen Beratung - professionelle und ethische Orientierung}

Die Arbeit der internen Beratung wurde in Leitlinien mit folgenden Grundsätzen beschrieben:

1. Systemische Orientierung meint, dass in der Arbeit mit dem Einzelnen oder der Gruppe ihre systemische Vernetzung in die Gesamtorganisation berücksichtigt wird. „Das Gesamte ist mehr als sie die Summe der einzelnen Teile“, der Synergieeffekt soll zum Ausdruck kommen (Ennen und Richter 2010). Außerdem berücksichtigt dies das Wirken der Wirklichkeitskonstruktionen, die von Einzelnen und Gruppen der Organisation vorgenommen werden. Methoden der systemischen Transaktionsanalyse (Schmid 2003; Mohr 2008) und der systemischen Organisationsanalyse (Mohr 2006, 2010) spielten deshalb in der praktischen Coaching-Arbeit eine wichtige Rolle.

2. Persönlichkeitsbezogenes Arbeiten hat zum Ziel, die Persönlichkeit der Teilnehmer von Maßnahmen zu entwickeln. Dies bezieht sich auf das Leben der Berufsrollen und ist dabei ganzheitlich auf ihr Verhalten, Denken, Fühlen und ihre Beziehungsgestaltung gerichtet. Modelltheoretisch sind darin die Rollentheorie (Schmid 2003; Mohr 2012) und die Ich-Zustandsvorstellung (Hagehülsmann und Hagehülsmann 2001; Mohr 2009) der ganzheitlichen Gestalt aus Denken, Fühlen und Verhalten zugrunde gelegt.

3. Ethik: Die Arbeit mit den Klientensystemen, egal ob es sich um Einzelne oder um mehrere Personen handelt, ist durch ethische Regeln geleitet. Dies gilt gerade heute in einer „Wirtschaft am seidenen Faden“ (Mohr 2012). So werden in der internen Beratung personenbezogene Informationen und Daten diskret behandelt. Der Diskretionsaspekt ist deshalb besonders hervorzuheben, da sich hier die interne Beratung von der Funktion Personalentwicklung in einem Unternehmen abgrenzt, die ja für die individuelle und unternehmensweite Personalplanung an Informationen interessiert ist.

4. Ressourcenschonendes Vorgehen und nachhaltige Entwicklung: Die knappste Ressource ist heute die Zeit. Maßnahmen werden deshalb so angelegt, dass eine angestrebte Veränderung ressourcenökonomisch erreicht wird. Alibi- oder Legitimationsveranstaltungen (,Schaut, wir haben doch irgendwas gemacht!“") werden in Unternehmen nicht selten angeregt. Sie schaden aber der nachhaltig positiven Entwicklung und sollten nicht durchgeführt werden. Dies verbietet z. B., Führungskräfte und Mitarbeiter in Präsenz-Veranstaltungen lediglich „unterzubringen“. Gerade bei Projekten, die eine größere Anzahl von Menschen betrifft, ist deshalb eine entsprechende „Architektur“" aus Einzelgesprächen, vor Ort zu erledigender Aufgaben, Klein- und Großgruppenmaßnahmen zu planen, die insgesamt die Entwicklung voranbringen.

5. Ständige Qualitätsverbesserung beinhaltet die Forderung, stetig die Qualität seiner Maßnahmen zu verbessern. Dies bedeutet in erster Linie ständige Weiterbildung und Supervision für die im Referat Tätigen, d.h. diese Anforderung wird an alle gestellt, die in der Beratung arbeiten. Außerdem trägt die regelmäßige Auswertung der Maßnahmen zur Qualität bei. So sind auch die Diagnostik der Folgen und die Evaluation des Transfers stetige Prozesse der Beratungsarbeit. 
6. Einheit von Diagnose und Intervention: Schon der Erstkontakt mit dem Kunden ist gleichzeitig diagnostische Information wie auch eine Intervention. Die Beziehung, die der Berater mit dem Kunden eingeht, wird von Anfang an als eine Veränderungsbeziehung konzipiert. Selbst wenn jemand sein Verhalten nicht ändert und sich nur seine Richtung bestätigt, wird zumindest in der Einschätzung oder Einstellung etwas verändert. Es geht darum, wie de Shazer (1990) es formulierte, Kunden statt Besucher oder Klagende zu haben oder sogar Co-Therapeuten, wie Schmidt (2005) ergänzte. Coaching ist durch sein Wirken in der Kommunikation geprägt. So lässt der Coach i. d. R. keine Abwertungen anderer Personen, anderer Bereiche oder der ganzen Firma (Mohr 2008) im Gespräch ohne Intervention durchgehen. Dies beginnt aufgrund üblicher Vorurteilsstrukturen in der Organisation („Die aus der Verwaltung sind ja ..."; „Es ist doch so, der Vertrieb verdient das Geld ..."). Auch Bemerkungen, die mit „In unserem Hause ist es doch so, dass...." beginnen, enthalten meistens Abwertungen im Sinne der Ausblendung von Möglichkeiten. Hier ist v. a. eine spezifische innere Distanz des internen Coachs seinem Unternehmen gegenüber nötig, die er sich z. B. durch kontinuierliche externe Supervision erhalten kann. Natürlich kann es bzgl. der Konfrontation von Abwertungen aus diagnostischen oder Interventionsgründen zeitweise eine Ausnahme geben. Aber die grundlegende Orientierung ist, keine verdeckte Kommunikationsebene zu etablieren, die Abwertungen zulässt. Dies setzt sich bei jedem Beratungskontakt fort. Die Steuerung der Beratung findet dabei aus dem Prozess der Beratung heraus statt. Welche Maßnahmenetikettierung vorgenommen wird, hängt von der Diagnostik und der Auftragsklärung ab. Diese Betonung ist wichtig, da die Klienten oft schon mit bestimmten Vorstellungen über die Maßnahmen kommen, ohne dass diese professionell diagnostisch abgeklärt wurden. Gerade in der internen Beratung ist dieser Aspekt wesentlich, weil gerne versucht wird, interne Dienstleister zu Erfüllungsgehilfen von vermeintlich wichtigeren Unternehmenseinheiten zu machen. Zu Beginn einer Maßnahme findet zunächst eine eingangsdiagnostische Abklärung statt, welche Maßnahme für die vorliegende Fragestellung sinnvoll ist. Dann wird in gemeinsamer Vereinbarung mit dem Kunden die Vorgehensweise beschlossen. Diese Ebene der gemeinsamen Vereinbarung ist ständiger Begleiter der Beratungsarbeit.

\section{Das Coaching-System im Einzelnen}

\subsection{Zusammensetzung der Gruppen}

Am Programm des internen Coachings in Gruppen nahmen 65 Führungskräfte der beiden unteren Führungshierarchie-Ebenen teil. Die Teilnahme war freiwillig und fand in geschlossenen Gruppen außerhalb der Kernarbeitszeit, meistens abends zwischen 17.00 und 19.00 Uhr, statt. Die Mitglieder einer Gruppe durften nicht in einer direkten organisatorischen Beziehung miteinander stehen. Die Gruppen wurden vom Coach nach den Erstgesprächen mit den Teilnehmern zusammengestellt, damit sie vom Erfahrungshintergrund her etwa gleich waren und von anderen Aspekten (Temperament, Geschlecht) eine gute Mischung zum gemeinsamen Lernen bildeten. 
Aus Seminaren mit unterschiedlichen Hierarchie-Ebenen gibt es die Erfahrung, dass ranghöhere Führungskräfte sich selbst unter Druck setzen, in ihrem Führungswissen „weiter“ zu sein, was nicht immer der Fall ist. Insofern gehörten die Gruppenmitglieder jeweils einer vergleichbaren Hierarchieebene an. Man hätte auch unterschiedliche Ebenen zusammennehmen können, um z. B. für die Unternehmenskultur wichtige hierarchieübergreifende Elemente oder Elemente des vertikalen Teams (Schmid und Messmer 2005) zu modellieren. Dies hätte allerdings die Komplexität der Gruppen und damit auch der Coaching-Interventionen deutlich erhöht und wurde deshalb nicht realisiert. Nun zunächst ein Beispiel für einen Einblick ins Detail:

Spätnachmittags pünktlich um 17.00 Uhr treffen sich fünf Führungskräfte zur InhouseCoaching-Gruppe „Führung und Management“, die jetzt 7 Monate im 4-Wochen-Rhythmus für jeweils zwei Stunden zusammenkommt, mit einem Coach im Bildungszentrum des Unternehmens.

Am Anfang stellt der Coach einige Fragen: ,Welche Resultate haben die Entscheidungen und Vorhaben vom letzten Mal gezeigt? Was beschäftigt Sie zurzeit in Ihrer Führungsrolle, was beschäftigt Ihre Mitarbeiter? Gibt es ein Anliegen, das Sie heute ausführlicher besprechen wollen?" Durch die Anfangsrunde werden auch die weiteren Themen für Coachings ermittelt. Heute wird Folgendes genannt:

- Einer von den Führungskräften will erfahren, wie er von Teamgesprächen, in denen er bisher fast ständig in der Rolle des Alleinunterhalters ist, hin zu mehr Beteiligung und Verantwortungsübernahme der Mitarbeiter kommt.

- Ein anderer hat in den nächsten Tagen ein schwieriges Gespräch mit einem Mitarbeiter zu führen, für das er Anregungen braucht.

- Ein dritter möchte gerne wissen, wie er den jüngsten Controlling-Bericht in seiner Vertriebseinheit interpretieren kann und wie er mit der Rückmeldung seines Chefs, er sei ,einfach zu zahm“, umgehen kann.

- Zusätzlich steht heute ein Theoriethema auf dem Plan: „Zeitstrukturierung unter Beziehungsaspekten“" (Mohr 2010, 52 ff.). Es nimmt mit Übung etwa 1/2 Stunde in Anspruch.

Anschließend erfolgt die Zeitplanung, und mit passenden Methoden werden die vorgestellten Fallsituationen mit einem Lerneffekt für alle bearbeitet, aber auch mit entsprechenden persönlichen Anregungen für den Fallgeber. Dabei steht die Stärkung der Selbstorganisationsfähigkeit der einzelnen Führungskraft im Vordergrund. Kurz vor 19.00 Uhr gibt es eine Auswertungsrunde, ein neuer Termin wird vereinbart und die Teilnehmer nehmen einen aktuellen Artikel über Wettbewerbstheorie zur Lektüre mit.

\subsection{Um welche konkrete Form von Coaching handelte es sich?}

Die Teilnehmer kamen nicht auf Weisung der Firma, sondern freiwillig in die CoachingGruppen. Für die geschlossene Coaching-Gruppe gab es einen Vertrag für ein Jahr, der die Teilnahme an allen - bei Einrechnung von Urlaubszeiten i.d. R. - zehn Sitzungen ausmachte. Abwesenheit war mit Entschuldigung aus triftigen Gründen möglich. In der Praxis ergab dies eine unterschiedliche Anwesenheitsquote in den einzelnen Gruppen. Dieses Verhalten gab mir manchmal zu denken, hatte aber - wie unten gezeigt wird - 
bestimmte Auswirkungen. Das Design der einzelnen Veranstaltungen der Coaching-Maßnahme bestand aus vier Elementen: 1) aktuelles Rollenstatement mit erstem Feedback, 2) ein Input aktueller Managementthemen, 3) Besprechung konkreter Fallsituationen aus dem Arbeitsalltag, 4) Abrundung in kleiner Abschlussrunde.

Diese Elemente bieten gerade durch ihre klare Struktur vielfältige Möglichkeiten der Ausgestaltung. Ausgehend von der Erfahrung, dass viele als „kollegiale Beratung von Peers" konzipierte Modelle nach einiger Zeit auslaufen, beanspruchte das Modell dieser Coaching-Gruppen eine größere Nachhaltigkeit, indem auch die Unternehmenskultur beeinflusst werden sollte. Insofern sollte es auch einen gewissen Reiz haben (Berne 1979), damit es für die Teilnehmer interessant blieb. Dieser bestand nicht nur in immer neuen Inputthemen, was dem Ganzen einen kleinen Akademiecharakter gab, sondern z. B. auch in der Intervention ,Tournee“, dass wir mit den Gruppen die einzelnen Organisationseinheiten und Arbeitsplätze der Teilnehmer abwechselnd besuchten. Eine weitere reizvolle Intervention war das Zusammenbringen von zwei Gruppen zu einem Termin. Dies ergab kaum Reibungsverluste; im Gegenteil, die dann jeweils zu Beginn neu getroffene Verschwiegenheitsabmachung ermöglichte schnell eine wechselweise Beratung. Allerdings wollten die Gruppen dies nicht zur Dauereinrichtung machen, sondern immer wieder Termine in ,ihrer" Gruppe allein haben.

Wesentlich ist auch die klare Präsenz eines professionellen Coachs als Leiter der Gruppensitzungen. Vorherige Erfahrungen mit Netzwerkgruppen von Peers ohne einen unabhängigen Leiter waren nach enthusiastischem Start immer nach einer gewissen Zeit eingeschlafen. Ohne Zweifel sind Peerssupervisionsgruppen in sozialen Berufen oder Balintgruppen im Gesundheitsbereich ohne expliziten Leiter lange lebensfähig. Im Managementbereich sind Peergruppen nach meiner Erfahrung nicht so ermutigend.

1. Aktuelles Rollenstatement: Das immer zu Beginn von jedem Teilnehmer gegebene Statement zu den aktuellen Rollenthemen fokussierte vier Perspektiven: ein kurzer Rückblick auf das Gewordene nach der letzten Coaching-Sitzung, dann die Orientierung in das Hier und Jetzt, womit die Teilnehmer sich in ihrer Führungsrolle und auch ihre Mitarbeiter zurzeit beschäftigen. In der Anfangsrunde besteht bereits die Möglichkeit für ein von Respekt getragenes, persönlich-professionelles Feedback seitens des Coachs zur Entwicklung des Einzelnen, und welches Bild er gerade darstellt. Dies beinhaltet auch schon ein mögliches Reframing des Bezugsrahmens des Coachees.

Darüber hinaus konnte jeder Teilnehmer sein Anliegen anmelden, das er ausführlicher als Fallsituation oder als Frage behandeln wollte. Dies diente auch zur Einübung von Achtsamkeit an dieser Stelle und auch der Unterscheidung beider Perspektiven. Denn die Führungsrolle rückt manchmal andere Themen in den Vordergrund als die Mitarbeiterrolle, etwa wenn es um Anforderungen an die Mitarbeiter geht oder in Extremfällen um die Teilnahme an einer Arbeitskampfmaßnahme. Dann wird das Rollenthema der Führungskraft sehr deutlich. Hilfreich ist hier das Konzept der „Leitrolle“ des Organisationsberaters Erich Hartmann: Die Leitrolle kann im Rahmen der Organisationsrollen dann auch die Rolle des Vertreters des Arbeitgebers sein. Insgesamt wird damit eine Fokussierung auf die Rollen im Unternehmen gegeben. Dies schloss nicht aus, dass die Teilnehmer auch einmal etwas aus ihrem Privatleben berichteten, was für die Lebensstilintegration und die Work-Life-Balance bedeutsam war. Das 
Anfangsstatement war immer von der Frage begleitet, wie die aktuelle Aufgabenerfüllung und Zielerreichung aussieht. Dies geschah vor dem Hintergrund, dass es wenig Sinn macht, z. B. über marginale Befindlichkeiten oder Verständnisfragen von Teammitgliedern untereinander zu sprechen, wenn gerade die betriebswirtschaftliche Seite gefährdet ist. Insgesamt diente der erste Teil v.a. dazu, die aktuelle emotionale Haltung der Coaching-Teilnehmer zu erfassen. Das erste kurze Feedback gibt hier schon einen bestimmten Bezugsrahmen mit Interventionscharakter, sei es eine Anerkennung für die Leistung in einer Situation, ein Mitfühlen oder auch ein Angebot, dies ausführlicher zu behandeln. Dies konnte das Angebot beinhalten, aus einem Sachverhalt ein Fallanliegen im weiteren Verlauf der Sitzung einzubringen. Aber auch die Technik der verhaltensmodifikatorischen Verstärkung wurde hier genutzt, um Fortschritte seit dem letzten Mal zu unterstützen.

2. Input aktueller Managementthemen: Der Input bestand i.d. R. aus einem Kurzvortrag (bis $10 \mathrm{~min}$. ) zu Diskussionen in der Managementliteratur oder für die Teilnehmer wichtige wirtschaftliche Trends mit entsprechender Übung oder Diskussion (20 min.). Dadurch haben die Teilnehmer innerhalb von zwei Jahren wesentliche Grundlagen der Führungspsychologie kennengelernt. Wenn sehr viele Coaching-Anliegen geäußert wurden, konnte der Inputteil auch einmal auf die nächste Sitzung verschoben werden, weil das Coaching im Vordergrund stand.

3. Besprechung konkreter Fallsituationen: Die Fallarbeit war als Coaching durch den Coach unter Einbeziehung der anderen Teilnehmer konzipiert. In Ausnahmefällen haben auch in Coaching weitergebildete erfahrene Führungskräfte unter Supervision des Coachs einzelne Coaching-Einheiten innerhalb der Gruppe durchgeführt. Dieser Teil hatte mit einer Zeitspanne von bis zu 90 min den größten Raum.

4. Abschlussrunde: Die Abschlussrunde ließ die Teilnehmer noch einmal ihre wesentlichen Punkte, die sie aus der Sitzung mitnehmen, fokussieren und in der Gruppe benennen. Für den Coach bietet sich dabei noch eine Interventionschance, wenn jemand etwas Wesentliches vergisst oder noch eine Konkretisierung nötig ist.

\subsection{Zwei zentrale Prozesskonzepte für die Coaching-Gruppen}

Das erste Prozesskonzept, das die Arbeit der Coaching-Gruppen bestimmt, ist das VierPhasen-Modell des Neuseeländers Hewitt (1995). Es dient der Strukturierung einer einzelnen Coaching-Einheit und beginnt mit einer Kontaktphase (Contact), die mit dem „Vertrag“, der Vereinbarung über die Vorgehensweise endet. Darauf folgt die Inhaltsphase (Content), die mit einer Entscheidung für einen bestimmten Fokus (decision) abschließt. Diese Neuentscheidung kann sich auf Verhalten, Denken, Gefühle oder auch auf das Selbstbild beziehen. Dann folgt die Konsolidierungsphase (consolidation), die die getroffene Entscheidung operationalisiert, für und in der Realität Umsetzungsmöglichkeiten entwickelt und überprüft. Die Phase endet mit einer Integration des Neuen. Das Intervallprinzip mit Sitzungen alle vier Wochen bot dafür eine gute Umsetzungsbasis. Den Abschluss bildet die Auswertungsphase (Conclusion), in der die Resultate und ggf. Schlussfolgerungen daraus bestimmt werden (vgl. Abb. 1).

Ein zweites Modell ist gerade für die 13 Coaching-Gruppen mit jeweils vier bis sechs Mitgliedern geeignet. In den Gruppen wird nach dem Toblerone-Modell (Schmid 2003; 
Abb. 1: Vier-Phasen-Modell von Hewitt (1995)

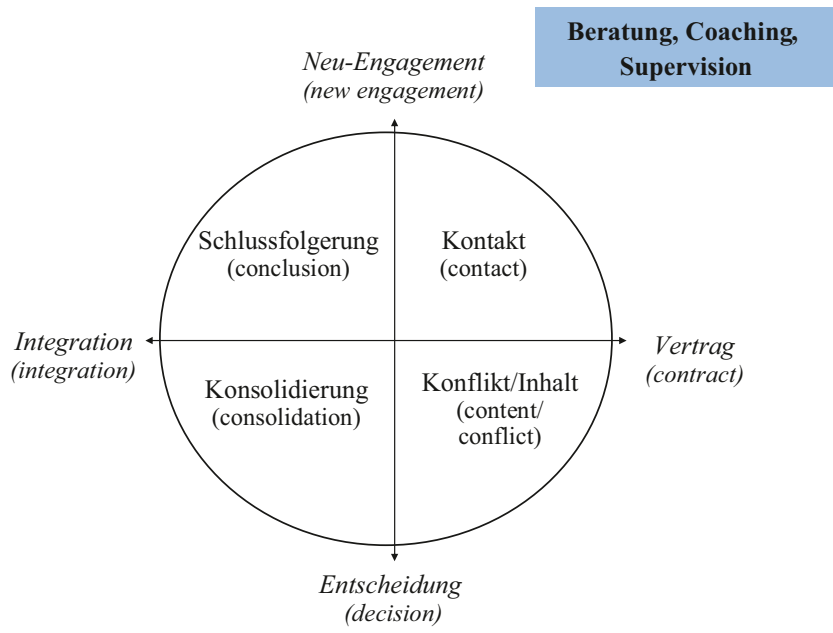

Mohr 2008) gearbeitet, was bedeutet, dass von Coaching-Einheit zu Coaching-Einheit die Gruppenmitglieder einen Zuwachs an Kompetenz in Führungs- und Vertriebskompetenz, in Theoriewissen und im praktischen Erfahren bekommen. Das „Toblerone“-Modell von Bernd Schmid veranschaulicht Bestandteile eines guten Lernprozesses. Es analogisiert die Toblerone-Dreiecksrippen-Schokolade aus der Schweiz mit dem Lern- und Entwicklungsprozess. Die drei Ecken mit guten Zutaten für den Lernprozess zur Professionspersönlichkeit sind folgende:

Feldkompetenz: Die Zeit, in der Fachkompetenz abgewertet wurde, ist vorbei: Zumindest die Bereitschaft und Fähigkeit, sich rasch in ein neues Fachgebiet einzuarbeiten, ist sehr wichtig. Feldkompetenz ist angewandte Fachkompetenz. Hier haben v. a. auch Austausch und Sharing der Erfahrung der Teilnehmer untereinander einen großen Stellenwert.

Theoriewissen im Soft-Fact-Bereich: Interessanterweise antworten Führungskräfte schon in einer früheren Untersuchung (Mohr 2008), dass ihnen im Coaching vermitteltes Theoriewissen zu Führung (z. B. Zur Bonsen 2010; Heinloth 2011), Kommunikation usw. entscheidend geholfen habe. Man könnte meinen, das ist eigentlich logisch, weil man ja auch in anderen professionellen Fachgebieten ebenfalls Theoriewissen verlangt. Und Führungskraft ist ein eigener zusätzlicher Beruf. Merkwürdigerweise wird in Unternehmen oft erwartet, dass man „Führen“ nebenbei lernen könne; allenfalls werden einige Konzepte und Techniken vermittelt. Viele Führungskräfte berichten, dass sie manche Konzepte erst Jahre, nachdem sie sie zum ersten Mal gehört haben, verstanden hätten. Und das Verstehen geht dann noch weiter. Immer wieder werden neue Facetten eines Zusammenhangs verstanden. Theoriewissen dient offensichtlich bei vielen auch dazu, das eigene Selbstbild zu klären. Es ist eine Grundillusion der Menschen, zu glauben, sie kennen sich selbst. 
Abb. 2: Das Toblerone-Modell von Schmid (2003)

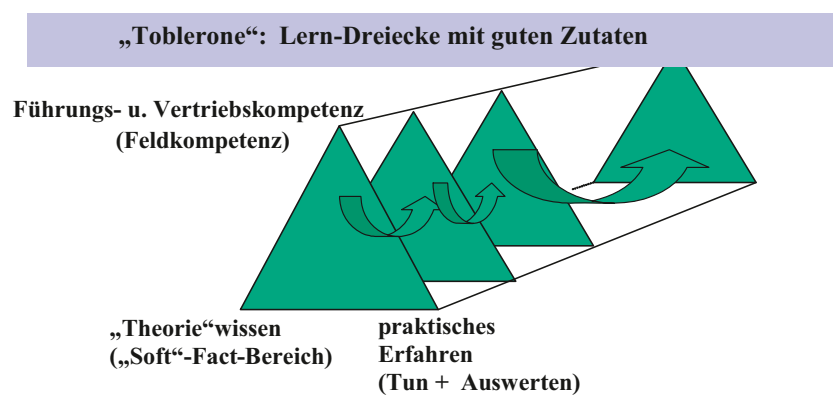

Praktisches Erproben (Tun und Auswerten): Manche Menschen waren schon sehr oft in einer bestimmten Situation, aber sie haben keine Erfahrung gewonnen. Erfahrung gewinnt der, der aktiv auswertet, wie es gelaufen ist.

Die drei Formen Feldkompetenz in Führung und Vertrieb, Theoriewissen im Soft-factBereich und das Tun und Auswerten stellen wichtige Elemente des VertriebsführungsCoachings dar (vgl. Abb. 2).

\subsection{Zwei zentrale Inhaltskonzepte für die Coaching-Gruppen}

Hinter dem hier vorliegenden Coaching-Ansatz stehen in inhaltlich-konzeptioneller Hinsicht Konzepte der systemischen Transaktionsanalyse (Mohr 2008) und der systemischen Organisationsanalyse (Mohr 2006). Als Ansatz der systemischen Transaktionsanalyse sei das Rollenweltenmodell (Schmid 1994) genannt, das in seiner neuesten Fassung fünf Rollenwelten beinhaltet (Mohr 2012):

Die Teilnehmer bekommen ein Bewusstsein über den Unterschied von Person und Rolle sowie über ihre Rollen in den Welten Unternehmensorganisation, Berufsqualifikation, Privatwelt und Gemeinwesenwelt. Damit war das Coaching durchaus nicht verengt auf reine Business-Skills, sondern umfassend auf die Entwicklung der persönlichen Professionalität im Sinne von „Life-Coaching“ (Buer und Schmidt-Lellek 2008; Schmidt-Lellek und Buer 2011) konzipiert. Insbesondere lernen sie für die Unternehmensorganisation und die Berufsqualifikation ihre Rollen bewusst professionell wahrzunehmen und weiterzuentwickeln. In den meisten Coaching-Fällen steht die Selbststeuerung der Führungskraft mit dem Aspekt der Integration all ihrer Rollenwelten im Vordergrund. Wenn eine Perspektive langfristig nicht bedient wird, entstehen Probleme.

Auch die eigene Konsumentenwelt ist gerade für Vertriebsmitarbeiter nicht uninteressant. Kauft er und oder würde er die Produkte seines Unternehmens selber kaufen? Oftmals bekommt man an dieser Stelle eine verneinende Antwort. Dabei ist nicht immer eine vollkommene Identifizierung mit jedem Produkt des Unternehmens nötig, aber eine völlige Abstinenz aus Einstellungsgründen ist nicht sehr zuträglich (vgl. Abb. 3).

Aus der „Systemischen Organisationsanalyse“ (Mohr 2006) steht v. a. das Modell der zehn Systemdynamiken Pate beim Coaching der Führungskräfte. Mit dessen Hilfe ist es möglich, jede Organisationseinheit bzgl. ihrer momentanen Performance zu analysieren und Schritte zur Entwicklung und weiteren Verbesserung einzuleiten. Hier verbinden sich auch die eher betriebswirtschaftlich orientierte Managementrolle und die MenschenFührungsrolle. Insofern bietet dieses Tool in der konkreten Coaching-Arbeit immer die 
Abb. 3: Das Rollenweltenmodell nach Schmid (2003) und Mohr (2012)

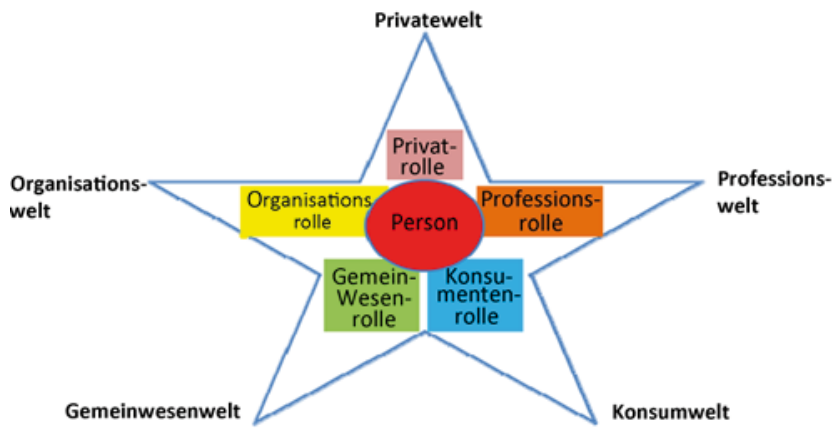

Möglichkeit zur Bestandsaufnahme. Es ermöglicht, die Organisationseinheiten der Führungskräfte immer wieder auf ihre Potenziale hin $\mathrm{zu}$ untersuchen und zu unterstützen (vgl. Tab. 1).

\section{Die empirischen Ergebnisse}

\subsection{Auswirkungen auf Verantwortungsübertragung und Beförderung}

Um herauszufinden, ob die Teilnahme an den Coachinggruppen einen Einfluss auf das berufliche Fortkommen der Teilnehmer hatte, wurde zunächst die Analyse mit der QCA (Qualitative Comparative Analysis) von Ragin (1987, 2000, 2008) durchgeführt. Die QCA ist ein algorithmisches Verfahren, das entwickelt wurde, um eine Methode zwischen Fallstudie und klassischen Kovarianzanalysen zu haben, die auch bei begrenzten Fallzahlen Aussagen über hinreichende und notwendige Bedingungen macht. Sie ermöglicht in sozialwissenschaftlichen, etwa auch organisationalen Zusammenhängen, Konfigurationen von unabhängigen Variablen zu ermitteln, die zu einer bestimmten Ausprägung der abhängigen Variable führen. Dabei ist auch eine Äquifinalität möglich, d.h. mehrere unterschiedliche Konfigurationen von unabhängigen Variablen führen zu einem bestimmten Ergebnis (Fiss 2007). Wie Katz und Kahn (1978, S. 30) in ihrem Standardwerk „The social psychology of organizations“ betonten: ,a system can reach the same final state, from different initial conditions and by a variety of different paths" (zit. n. Fiss 2007, S. 1181). Auf dieser Grundlage findet die QCA als interessante empirische Methode eine zunehmende Anwendung im organisationstheoretischen und wirtschaftswissenschaftlichen Bereich (Jackson 2005; Bentrop und Schneider 2012).

Die QCA wurde von Ragin (1987, 2000, 2008) auf Basis der Booleschen Algebra entwickelt und beruht konzeptionell auf mengentheoretischem Denken. Der darauf aufbauende Algorithmus ermöglicht Aussagen zu sowohl hinreichenden als auch notwendigen Bedingungen. Man bekommt Aussagen darüber, wie deutlich das Auftreten bestimmter Ausprägungen der unabhängigen Variablen auch bestimmte Werte der abhängigen Variablen nach sich zieht, genauso wie die Abwesenheit bestimmter Ausprägungen der unabhängigen Variablen zum Nichteintritt der Werte der abhängigen Variable führt. Die QCA wurde hier als fsQCA (fuzzy set Qualitative Comparative Analysis) durchgeführt (Wagemann und Schneider 2007). Dies bedeutet, dass die aufgetretenen Situationen der 
Tab. 1: Das Modell der zehn Systemdynamiken (Mohr 2006)

\begin{tabular}{lll}
\hline Dynamik-Felder & Die zehn Systemdynamiken & Einzelfragen zu den Dynamiken \\
\hline System-Struktur & Dynamik der Aufmerksamkeit & $\begin{array}{l}\text { Womit beschäftigen sich die Leute in } \\
\text { der Organisation (-seinheit) zurzeit am } \\
\text { meisten? }\end{array}$
\end{tabular}
meisten?

Dynamik der Rollen

Dynamik der Beziehungen

System-Prozesse Kommunikationsdynamiken

Problemlösedynamiken

Erfolgsdynamiken

System-Balancen Dynamik der Gleichgewichte

Dynamik der Rekursivität

System-Pulsation Dynamik, „Äußere System-Pulsation" (Äußere Grenzlinien, Offenheit, Geschlossenheit)

Dynamik „Innere System-Pulsation" (Innere Grenzlinien, Subsysteme)
Wie verhält sich das, was im Moment die Hauptaufmerksamkeit genießt, zu dem, was eigentlich Ziel der Einheit ist?

Welche Rollen gibt es momentan im System?

Welche Merkmale haben die Rollen?

Verändern diese sich zurzeit, und wenn ja, dann wie?

Wie stehen die Rollen und die Personen miteinander in Beziehung?

Welche Grundbotschaften gibt es zwischen den Rollenakteuren?

Was charakterisiert die Art, wie man miteinander kommuniziert?

Was sind zurzeit „Probleme“?

Wie geht man damit um?

Wie erreicht oder vermeidet man Erfolge?

Welches Gleichgewicht würde wer gerne erhalten?

Welches Gleichgewicht wird angestrebt?

Wie sind ähnliche Prinzipien auf unterschiedlichen Ebenen der Organisation verwirklicht?

Wie entwickelt sich zurzeit die äußere Grenzlinie des Systems?

Welche Maßnahmen braucht es, um eine ,angemessene“" Offenheit und Geschlossenheit herzustellen?

Welche relevanten Subsysteme lassen sich in der Organisation zurzeit unterscheiden und wie wirken sie sich aus?

unabhängigen und der abhängigen Variablen in einer bestimmten Abstufung metrisch auf ein Spektrum zwischen 0 und 1 skaliert werden.

Die unabhängigen Variablen waren die Dauer der Teilnahme in der Coaching-Gruppe sowie die Anwesenheitsquote an den Gruppensitzungen. Die Teilnahmedauer unterschied sich in „,fast keine Teilnahme“, also Leute, die nur kurz „hineinschnupperten“ und dann 
die Gruppe wieder endgültig verließen, über Teilnehmer mit kurzer Teilnahmezeit (bis $1 \mathrm{Jahr}$ ), über Teilnahme bis zu drei Jahren und über drei Jahre. Die Anwesenheitsquote bemisst sich aufgrund der freiwilligen Teilnahme und der Regel, dass man durchaus bei zeitgerechter Entschuldigung aufgrund triftiger Gründe auch einmal absagen durfte, in einem sechsstufigen Spektrum von geringer Anwesenheit, über vorhandene Anwesenheit mit größeren Lücken, deutliche Anwesenheit mit erkennbaren von außen bedingten Gründen (2-3 von 10 Sitzungen im Jahr), über nur geringe Abwesenheiten bis hin zu nahezu vollständiger Anwesenheit. Interessant war, dass sich hierin die Gruppen in ihren Kulturen und damit vielleicht auch ihrem inneren Verpflichtungsempfinden sehr deutlich unterschieden. Dieser Gruppeneffekt wurde aber hier nicht betrachtet.

Die untersuchte resultierende Variable war die „Übertragung von zusätzlicher Verantwortung durch die Firma“. Diese Verantwortungsübertragungsentscheidungen werden völlig unabhängig von den Coaching-Gruppen und aufgrund der Schweigepflicht des Coachs auch explizit ohne seine Expertise von Management und Personalabteilung in der Firma getroffen. Bei diesem Kriterium wurden drei Stufen gewählt. Die höchste Ausprägung bestand in einer klassischen Beförderung auf eine höhere Führungsposition mit höherer Hierarchieebene, mit mehr Mitarbeitern und mit höherer Bezahlung (höchster Wert). Eine etwas niedrigere Einstufung bekam die Übertragung einer Führungsaufgabe auf der gleichen Hierarchieebene, aber mit signifikant höherer Mitarbeiterzahl (mindestens plus 50\%) und i.d. R. damit verbundener höherer Bezahlung. Auch die zusätzliche Übertragung einer wichtigen Projektaufgabe oder Zusatzrolle (etwa Mentor oder Vertriebs-Coach) wurde gewertet. Niedrige, aber vorhandene Wertung bekam dann noch die offizielle Übertragung der Verantwortung aus einer bisher kommissarischen Rolle in eine feste unbefristete.

Als Ergebnis zeigte sich, dass insbesondere die Anwesenheitsquote, also regelmäBige kontinuierliche Teilnahme in den Coaching-Gruppen mit deutlicher und bei höherer Anwesenheit wesentlich höherer Verantwortungsübertragung zusammenhängt. Der Konsistenzwert, der im Bereich 0 (=keine Konsistenz) bis 1 (=vollständige Konsistenz) liegen kann und eine Aussage über den Zusammenhang zwischen unabhängigen und resultierender Variable angibt, beträgt für beide unabhängigen Variablen zusammen 0,82. D.h. der Zusammenhang ist recht deutlich, selbst wenn man für einen aussagefähigen Wert einen hohen Maßstab, etwa 0,75 unterstellt (Wagemann und Schneider 2007). Der „Coverage“-Wert, d.h. die Anzahl der Fälle, die er erklärt, ist mit 0,85 auch recht hoch. Auch bzgl. der Einstufung als notwendige Bedingung ergibt sich, dass bei Teilnehmern ohne deutliche Anwesenheitsquote mit einer Übertragung von Verantwortung kaum zu rechnen ist.

Die Dauer der Teilnahme hat eine etwas schwächere Aussagekraft. Zu etwa $70 \%$ kann man davon ausgehen, dass Dauergäste in einer Coaching-Gruppe auch mindestens einmal oder sogar mehrfach zusätzliche Verantwortung übertragen bekommen. Als notwendige Bedingung kann man sagen, dass das Fehlen von Dauer und Anwesenheit auch bei Teilnehmern einer Coaching-Gruppe der sichere Weg zur Nichtbeförderung ist.

Die jeweilige Anwesenheitsentscheidung, die zwar durch die geschlossenen Gruppen und den Zwang zur Entschuldigung mit triftigem Grund ein wenig eingeschränkt war, offenbart sich durch ihre unterschiedliche Handhabung im Nachhinein als eine interessante Größe. Sie ist eng mit einer davon völlig unabhängigen Entscheidung durch Dritte, 
nämlich der Verantwortungsübertragung verbunden. Das heißt Leute, die sich selbst immer wieder mit Themen der Weiterentwicklung im Coaching befassen und dafür auch für andere da sind, werden offensichtlich eher für höhere Verantwortung entdeckt als die, die das nicht tun. Die Gesamtdauer der Teilnahme spielt auch eine Rolle. Aber wenn eine gewisse Mindestzeit (1 Jahr) überschritten war, ist sie nicht mehr so entscheidend.

Andere unabhängige Variablen wie Geschlecht (männlich, weiblich) oder ob jemand im Vertrieb oder im Betrieb arbeitet, wurden auch geprüft, hatten aber keinen signifikanten Einfluss auf die „Übertragung zusätzlicher Verantwortung; auch Kombinationen, etwa im Beispiel „weibliche Vertriebsmitarbeiterin mit hoher Anwesenheitsquote und Teilnahme zwischen ein und drei Jahre“, brachten keine höhere Wahrscheinlichkeit für Verantwortungsübertragung. Nach diesen allgemeinen Ergebnissen nun ein Blick in die Einzelheiten der Wirkungsbedingungen.

\subsection{Wirkungsbedingungen in Coaching-Gruppen}

Ende des Jahres 2010 führten wir außerdem eine Untersuchung zu Wirkungsbedingungen im installierten internen Coaching-System durch. Was wirkt in den Coaching-Gruppen? Damit hängt durchaus auch die Frage zusammen: Was lässt Manager ihre Freizeit über die Arbeitszeit hinaus für Coaching investieren? Dazu lagen mittlerweile die Erfahrungen mit dieser Arbeit über einige Jahre vor, die mit zwei Gruppen erfolgreicher Vertriebsführungskräfte begonnen hatte (Mohr 2000). Zu Beginn waren es in der Mehrzahl Frauen, die in Vertriebsführungspositionen erfolgreich waren und Interesse und Bereitschaft hatten, tatsächlich an ihren Kompetenzen zu arbeiten. Für viele Männer war es vermutlich noch zu bedrohlich, sich auf eine solch ,harte“ Form von Entwicklungsprozess einzulassen. Mittlerweile hat sich das Geschlechterverhältnis ausgeglichen.

$\mathrm{Zu}$ den Wirkbedingungen im Gruppen-Coaching hatten wir keine bestimmten Hypothesen. Deshalb wurden die Fragen an die Teilnehmer/innen auch sehr offen gestellt. Die Wirkungsbedingungen des Einzel-Coachings könnten mit den Bedingungen verwandt sein, wie sie Grawe (1994) für professionelle persönliche Entwicklungsunterstützung, etwa auch Therapie, untersucht hatte. Er fand vier zentrale Faktoren heraus: Aktive Hilfe zur Problembewältigung, Problemaktualisierung der realen Erfahrung, Klärungsarbeit oder Klärung, Ressourcenaktivierung. Diese könnten auch für Coaching-Prozesse in Gruppen relevant sein, aber dazu später in der Diskussion. Aber welche Wirkbedingungen werden für Gruppen-Goaching eine Rolle spielen?

Zur Durchführung der Studie: Die zu dem Zeitpunkt im System der Coaching-Gruppen befindlichen 65 Führungskräfte wurden mit einem Fragebogen offen befragt, welche Aspekte für sie beim Coaching wichtig waren. Der Rücklauf war sehr gut. Im kurzen Befragungszeitraum von drei Wochen lagen die differenzierten Antworten von 38 Teilnehmern, d.h. $60 \%$ der Gesamtteilnehmerzahl, vor. Insgesamt gab es 106 differenzierte Antworten mit 403 genannten Einzel-Items, mit denen die Teilnehmer die erfahrenen Effekte der Coaching-Gruppen beschreiben. Eine Inhaltsanalyse (Berelson 1952; Mayring 2010) wurde mithilfe von Frequenzanalyse und Valenzanalyse durchgeführt. Die Frequenzanalyse bezieht sich darauf, wie häufig bestimmte Begriffe genannt werden, die 
Valenzanalyse darauf, mit welcher Bewertung die Begriffe erwähnt werden. Sie ergab folgende Resultate.

\subsection{Die Ergebnisse}

Das erste und grundlegende Ergebnis war: Die Antworten zeigten sich sehr individuell, wie überhaupt die für das Coaching so zentrale individuelle Orientierung und damit auch die individuelle „Output-Ernte“ hervorgehoben werden. Nur wenige Themen werden häufiger erwähnt und auch durchaus nicht so sehr häufig. Dabei wird eine Beziehungsund Systemvariable, nämlich die Art des Zusammenarbeitens in 24,5\% der Antworten angesprochen. Fügt man alle Begriffe zusammen, die „Miteinander, Zusammenarbeit, gegenseitige Unterstützung, Team, Netzwerkbildung und Gruppe“ erwähnen, bekommt dieser Bereich die deutlichste Betonung. Die zweithäufigste Erwähnung bei den GesamtItems fanden mit 19,8\% Führungsaspekte, was bei einem Gruppen-Coaching für Führungskräfte nicht verwundert, aber dennoch Hinweise für die Fokussierung solcher Maßnahmen gibt.

Analysiert man alle Antworten differenziert nach inhaltsanalytischen Kriterien nach Feldern, so lassen sich sechs verschiedene Bereiche identifizieren: 1) Grundkonzeption der Coaching-Gruppe, 2) Themen, 3) Rahmenbedingungen, 4) Rollenspezifische Outputs und Resultate, 5) Outputqualität und Form der Umsetzbarkeit sowie 6) System- und Unternehmenskultureffekte. Im Folgenden werden die Ergebnisse in diesen Clustern qualitativ aufgeführt.

1. Grundkonzeption der Coachinggruppe: Die Grundkonzeption und Form des Arbeitens in der Coaching-Gruppe wird von 30,22\% der Teilnehmer in ihren Aussagen thematisiert. Als wesentlich wird dabei eine professionelle Grundausrichtung betont, die das Lernen für die Praxis ermögliche. Dazu gehöre die theoretische Fundierung des Vorgehens, die auch durch den Coach immer wieder gekennzeichnet werde. Die angewandten Methoden würden belegt und erklärt. Die bestehenden Angebote für gezielte Einzel-Coachings seitens des Moderators sowie aus Teilnehmersicht „,neutrales“ Feedback und Hilfestellung für diverse Themen kennzeichne das professionelle Vorgehen. „Da teilweise sehr vertrauliche Themen besprochen werden, ist ein entsprechendes Vertrauen zu den Mitgliedern der Gruppe erforderlich und auch gegeben“, gibt ein Teilnehmer zu bedenken. Zuverlässigkeit, Vertrauen, Verschwiegenheit, Diskretion und Offenheit sind - laut Teilnehmern - Kennzeichen der Coaching-Gruppe. Sehr viele Antworten bezogen sich auf den Prozess des Arbeitens in den Gruppen. Dabei wird die Neutralität und Unabhängigkeit des Coachs als wichtiger Faktor angesprochen. Prozesselemente des Vorgehens im Coaching spielen also eine große Rolle. So wird bspw. als wichtig erachtet, immer wieder Wahlmöglichkeiten des Handelns zu erhalten. Eine Anpassung der Lösungstiefe an den Bedarf finde statt, z. B. Simulation wenn nötig, also kein schabloniertes Vorgehen, dass z. B. bei einer bestimmten Frage immer ein bestimmter Standardablauf abgespult wird, wie etwa immer „Gespräche darüber" oder immer eine praktische Rollensimulation, sondern eine Intervention nach Erfordernissen. Ressourcenschonendes, insbesondere zeitressourcenschonendes Vorgehen wird registriert (,schnell, effizient, sofort umsetzbare Arbeit"). Das Lernen 
findet auf einer praktischen und konkreten Ebene statt. Die Themen und das Vorgehen werden theoretisch fundiert behandelt.

2. Inhaltliche Themen: Die inhaltlichen Themen finden in 11,32\% der Antworten Erwähnung. Dabei ist Führung der am meisten gewählte Begriff. Die Thematisierung und Motivation zum Führen scheint wichtig zu sein. Denn nicht selten ist gerade für Leute mit Vielfachrollen, insbesondere auch einer Vertriebsrolle, die eigentliche Mitarbeiterführung ein notwendiges Übel, das aber durch ein entsprechendes lösungsorientiertes Forum auch Spaß machen kann. Dazu ein Zitat: „Stetige Anleitung zum Führen findet statt, die auf Vertrauen und Stillschweigen begründet ist und die Entwicklung der Führungskraft begleitet." Andere immer wiederkehrende Themen sind insbesondere durch die Vertriebsleute der Ziel- und Zahlendruck, der sich durch die Finanzkrise noch erhöht hatte, weil die Geschäfte mit den Kunden schwieriger geworden waren. Aber v. a. die individuellen drängenden „Themen meiner Organisationseinheit" und „meine eigenen besonderen Themen“" stehen im Vordergrund. Im Coaching und auch in seiner Unterform Gruppen-Coaching bekommt man etwas Individuelles. Das beinhaltet Lösungen für die eigenen Führungsaufgaben, aber auch die Veränderungsprozesse im Unternehmen. Die aktuellen Inputthemen halten außerdem auf dem Laufenden. Erwähnt wird in den Antworten, dass Situationen wie der Zahlendruck, der Zieldruck und Geschäftsstrategien, die in anderen Einheiten ähnlich aufgetreten sind, bedarfsorientiert bearbeitet werden. Genauso sei es wichtig, auch „Dampf ablassen“, „Frust von der Seele reden“ sowie „Luft ablassen“ bzw. auftanken zu können.

3. Rahmenbedingungen: Das von internen Personalentwicklern in Firmen für Führungskräfte oft als wichtig erachtete - und in entsprechenden Zufriedenheitsfragebögen gerne ermittelte - Umfeld in Form von angenehmen Bedingungen spielt für die Teilnehmer einer Coaching-Gruppe nur eine geringe Rolle. Rahmenbedingungen werden nur in 5,67\% der Statements erwähnt. Und dann beziehen sich die Aussagen auf die Mischung der Gruppen, die ja gesteuert wurde, aber auch die Freiwilligkeit der Teilnahme und die Aufwands-Nutzen-Relation, insbesondere was Zeit und Output betrifft. Äußere Bedingungen wie Räumlichkeiten oder Catering wurden in keiner Aussage erwähnt. Selbst die manchmal schwierige Parkplatzsituation am Bildungszentrum bot kein Grund zur Klage. Obwohl das Coaching in der Firma, wenn auch in dem etwas außerhalb gelegenen Bildungszentrum stattfand, wird es als ,außerhalb des alltäglichen Rahmens" erlebt.

4. Rollenspezifische Outputs und Resultate: Auf einzelne Rollen, insbesondere die Führungs- und die Vertriebsrolle bezogene Antworten machen 16,98\% aus. Insbesondere für das Thema Führung gibt es offensichtlich Input in den Gruppen, allerdings auch „Praktisches“ für das Thema Vertrieb, wie mehrfach hervorgehoben wird. In den Antworten kommt der gesamte Führungsprozess zum Ausdruck. Es beginnt damit, die Führungsrolle anzunehmen und zu gestalten. Aber auch das Zusammenspiel von Fach- und Führungsrolle sowie die eigene Rolle im Gesamtunternehmen werden erwähnt. Modernes entwicklungsorientiertes Führen, eine Führungskonzeption ein- und umzusetzen und das Kennenlernen von Führungstechniken, die direkt auf das Bedürfnis des Einzelnen abgestimmt sind, charakterisieren weitere rollenbezogenen Ergebnisse. Personalentwicklung sowie betriebswirtschaftliches Denken 
und Handeln unterstützen dies. Zur Rollenkompetenz gehören das Lernen von Stressbewältigung, Problemlösung und eine bessere Einschätzung der eigenen Persönlichkeit. Es beinhaltet Führungstheorien umzusetzen, konkrete Gesprächssituationen für schwierige Mitarbeitergespräche zu strukturieren und ggf. auch zu simulieren. Ideen zur Mitarbeiterführung und zum Umgang mit schwierigen Mitarbeitern und Kunden sowie zur besseren Ausrichtung meiner Organisationseinheit sind rollenspezifische Outputs.

5. Outputqualität und Form der Umsetzbarkeit: Insgesamt 15,09\% der Antworten beziehen sich wertend (Valenzanalyse) auf die Outputqualität. Allgemeine Outputs werden in 11,32\% der Antworten thematisiert. Ganz einfach werden zunächst Stichworte wie Hilfe, Tipps und Anregungen erwähnt. Die schon unter Form des Arbeitens genannten emotionalen „Ventileffekte“ („Dampf ablassen“) oder Klärungen sind hier auch zu erwähnen. Im Einzelnen werden auch die Vorbereitung auf Situationen, aber auch Wissenszuwachs und theoretisches Rüstzeug aufgezählt. Es gebe sehr viel Aufbau und eher Neues, weniger Konservierung und Stabilisierung von Althergebrachtem oder etwa Rückschau auf glorreiche alte Zeiten. Der Fokus liege eindeutig im Entstehen von Neuem. Aktualität sei dabei ein weiterer wichtiger Punkt. Der kommt natürlich durch die von den Teilnehmern erlebten und eingebrachten aktuellen Anliegen zustande, aber auch durch das Einbringen von aktuellen Managementthemen durch den Coach. 3,77\% betonen noch andere Aspekte der Outputqualität. Es gebe schnell Realisierbares. Die Lösungsansätze seien neu. Das Eigene, Individuelle werde hervorgehoben; es gebe keine Standardlösungen, sondern solche, die auf den Einzelnen zugeschnitten seien. In der Praxis Umsetzbares und individuelle Problemlösung für Führungsthematiken werden hervorgehoben. Zitat: „Regelmäßiger Austausch wirkt sich positiv auf Belastbarkeit, Frustrationstoleranz und damit auch auf den Gesundheitszustand der Führungskräfte aus. “

6. System- und Unternehmenskulturvariablen: In diesem Bereich scheint für die Teilnehmer ein Schwerpunkt der Effekte zu liegen. 20,75\% der Antworten gehen auf Unternehmenskultur- und Systemeffekte ein. Dazu einige typische Zitate: „Netzwerke im Haus entstehen, die bei der täglichen Arbeit helfen, bestimmte Themen schneller und damit effizienter im Interesse aller Beteiligten zu lösen“. Man lernt, Wünsche und Probleme der anderen Bereiche zu verstehen. „Es entsteht ein besseres Verständnis füreinander, ein Wir-Gefühl im Unternehmen, das über die einzelne Organisationseinheit hinausgeht." Ein Effekt geht auch in die Privatwelt, es ist die „Entlastung der Privatpartner“. „Das eigene Thema kann so aus verschiedenen Blickwinkeln betrachtet werden, neue Vorgehensweisen können entstehen.“ Begleitung und Erprobung einer Kommunikation von Veränderungsprozessen (Aufbau neuer Team- und Gruppenstrukturen) wird durch das Gruppen-Coaching unterstützt. „Je besser die einzelne Führungskraft fortgebildet wird, desto höher ist im besten Fall die Qualität aller Mitarbeiter.“ 
Damit ergab sich folgendes Bild:

\begin{tabular}{lr}
\hline Charakteristiken der Wirkungsbedingungen & \\
\hline Grundkonzeption der Coachinggruppe & $30,22 \%$ \\
Spezifische Themen, die behandelt wurden & $11,32 \%$ \\
Rahmenbedingungen & $5,67 \%$ \\
Rollenspezifische Outputs & $16,98 \%$ \\
Outputqualität & $15,09 \%$ \\
Unternehmenskultur- und Systemeffekte & $20,75 \%$ \\
\hline
\end{tabular}

\section{Diskussion}

1. Zur Individualität der Ergebnisse: Diese Ergebnisse meiner Coachinggruppen ähneln den Erfahrungen, die man auch in der Kurzzeitberatung gemacht hat. So berichtete Steve de Shazer (1990) über seine Forschungen zur Arbeit Milton Ericksons, wohl eines der herausragendsten praktischen Psychotherapeuten des 20. Jahrhunderts. In dessen Vorgehensweisen konnte de Shazer mit seiner Milwaukee-Forschungsgruppe zunächst sieben Cluster von Strategien herausfinden. Es gab allerdings ein Problem, die siebte Kategorie, die Residualkategorie mit einer Vielzahl von nicht vergleichbaren Methoden, enthielt etwa $45 \%$ der Fallbeispiele. Also wurde die Kategorienzahl verdoppelt auf vierzehn. Das Resultat war, dass in der vierzehnten Kategorie, der neuen Residualkategorie, immer noch $35 \%$ der Fälle lagen. Wesentlich ist also die Einstellung auf die individuellen Muster. Genau diese individuelle Orientierung gehört auch zum Gruppen-Coaching in der internen Beratung.

2. Gruppe und Zusammenarbeit werden eindeutig hervorgehoben: Dies korrespondiert mit Ergebnissen aus anderen Feldern, z. B. der Coaching-Ausbildung, in der sich der Effekt der Gruppenkohäsion als außerordentlich wichtiger Faktor für den Erfolg der Weiterbildung herausgestellt hat (Rauen 2007). Die Arbeit in den Coaching-Gruppen ist aufgrund der zeitlichen Beschränkung sehr intensiv. Dies ist aber für aktive Manager, die eine solche Maßnahme quasi eingebettet in ihre tägliche Arbeit nutzen, eine offensichtlich sinnvolle Rhythmik. Im Gegensatz zu Mehrtages- oder Ganztagsveranstaltungen, in denen eine gewisse Entschleunigung, bestimmte Rituale des Ankommens, des Einfädelns usw. eine größere Rolle spielen, ist hier ein sehr ressourcenoptimierendes, effizientes Arbeiten gefordert. Dies wird aber von den Teilnehmern als sehr stimmig erlebt. Klassische Seminarrituale hätten dort Verwunderung ausgelöst.

3. Vertrauen und Diskretion in der internen Coaching-Gruppe: Interessant ist, dass mir in der ganzen Zeit der Gruppen - immerhin 10 Jahre - keine Indiskretion bzgl. persönlicher Informationen bekannt geworden ist, obwohl sicherlich etwa 120 Teilnehmer die Gruppen durchlaufen haben. Lediglich einmal ist mir zu Ohren gekommen, dass eine neue Teilnehmerin aus der Abteilung Öffentlichkeitsarbeit sich bei ihrem Chef über den Coach beschwert hatte. Der sei manchmal zu wenig „staatstragend“ 
und zeige nicht immer die Vorzüge des Unternehmens auf. In der nächsten Sitzung wurde sie von einem anderen Teilnehmer korrigiert. Er erläuterte ihr den Sinn und das Vorgehen des Coachings. Dieser Einzelfall war, wenn man die Sensibilität interner Systeme der Mitarbeiterförderung in der Praxis betrachtet, nicht unproblematisch, da Vertrauen schon durch eine einzige Entgleisung gefährdet sein kann. Außerdem war das System der Coaching-Gruppen auch auf die Unterstützung durch höhere Führungskräfte angewiesen. Diese wichtige Existenzgrundlage des Systems war durch den Vorfall gefährdert. Aber durch die direkte Korrektur des Teilnehmers ging dieser Vorgang gut aus. Damit zeigten die Coaching-Gruppen bereits einen positiven Unternehmenskultureffekt. Denn wenn ein solches internes Coaching-System zum Unternehmensbestandteil werden soll, muss es robust gegen Unverständnis von außen werden.

4. Aktive Hilfestellung, Problemaktualisierung, Klärung, Ressourcenaktivierung: Coaching ist aktive Hilfestellung, Problemaktualisierung, Klärung und Ressourcenaktivierung. Diese von Grawe (1994) hervorgehobenen Punkte kann man implizit aus den Antworten der Teilnehmer herauslesen. Sie sind also sicher auch enthalten. Allerdings werden sie in einem offenen Interview nicht so deutlich benannt. Der Bezugsrahmen, in dem die Teilnehmer selbst Wirkungsbedingungen sehen, ist anders. Insbesondere der Beziehungsaspekt der Art des Zusammenarbeitens, der gerade bei einer InhouseMaßnahme ein diffiziler Punkt ist, und auch die im hier vorliegenden Setting durchaus inkludierte pädagogische Seite zu Management- und Führungsthemen kommen deutlich hinzu.

\section{Spezifische Auswirkungen der Coaching-Gruppen}

1. „Das wichtigste Thema des Jahres “: Eine anonyme Befragung von Teilnehmern der Coaching-Gruppen für die Arbeit des Jahres 2006 zu einem früheren Zeitpunkt (Mohr 2007) ergab damals Ergebnisse zum Nutzen und zum Erleben dieses methodischen Ansatzes: Auf die Frage, inwieweit die Teilnehmer die Themen und Problemstellungen, die ihnen wichtig waren, im Gruppen-Coaching besprechen konnten, gaben $76,5 \%$ die maximale Wertung mit vollkommen ausreichend. Dass das Gruppen-Coaching für den Teilnehmer selbst bei den entscheidenden Herausforderungen im Jahr hilfreich war, beantworteten $94,1 \%$ mit ja. Darüber hinaus ergaben sich vielfältige andere Nutzen.

2. Verbesserung der Zusammenarbeit, Selbstmanagement, Lösungsorientierung, Gruppe: Es beginnt mit der Verbesserung der Zusammenarbeit mit den Mitarbeitern (Anregungen für das Verhalten gegenüber Mitarbeitern, heikle Themen mit Mitarbeitern anpacken) und setzt sich mit der persönlichen Entwicklung in Bezug auf das Selbstmanagement fort (souveräneres Auftreten, neue Gesichtspunkte in scheinbar verfahrenen Situationen, Gewinnung neuer Perspektiven bei Vorgehensweisen, Verminderung von Selbststress, Steigerung der Selbstachtung, Verdeutlichung der eigenen Rollenkompetenz, Zielgerichtes Vorgehen, Selbstkritik). Ein Großteil der Führungskräfte hob zudem die Lösungsorientierung hervor (Erarbeitung von Lösungen für konkrete Themen, Entwicklung verschiedener Lösungsstrategien unter Berücksichti- 
gung der eigenen Probleme sowie der Probleme der Mitarbeiter, Lösungsvorschläge, die zur Anwendung kommen). Somit zeigt sich insgesamt, dass der Nutzen nicht nur in dem inhaltlichen Einzelthema, sondern in der „Kultur“ des Erarbeitens gesehen wird. Fast alle hoben auch in der früheren Untersuchung die Art der Zusammenarbeit in den Gruppen hervor. Es fallen Stichworte wie: vertrauensvolle, offene und ehrliche Zusammenarbeit, Diskretion, optimale Teilnehmerzahl, homogene Gruppe, kommunikativer, kritischer Austausch. Weitere wichtige Punkte waren die Steuerung der Arbeit der Gruppen (vorrangige Behandlung besonders dringlicher Probleme, Frage nach aktuellen Fragestellungen, sofortiges Aufgreifen der Brennpunkte, Vereinbarung mit den Teilnehmern) und das selbstständige und gemeinsame Erarbeiten von Lösungen (Finden von Problemlösungen, selbstständiges Erarbeiten von Lösungen, Kombination von eigenen Themen, fremden Themen und Theorie, Unterstützung bei Entscheidungen, Anregungen, Hilfestellung für weitere Vorgehensweise).

3. Persönliche Entwicklung als Führungskraft: Es ergaben sich sechs Dimensionen des Nutzens: die eigene Persönlichkeit respektieren, Persönlichkeit von Mitarbeitern respektieren, krisenhafte Erscheinungen besser meistern, Feedback zu eigenem Verhalten bekommen, Umgang mit Veränderungsprozessen der Organisation, Rollenbewusstsein im Unternehmen als Manager und im Privatleben als Partner bzw. als Elternteil.

4. Perspektive der Systemqualifizierung: Hier wurden folgende Effekte beschrieben: mehr Bereitschaft auch der Mitarbeiter zur Weiterbildung, direkteres Ansprechen von Konflikten, Belebung der Kommunikation, mehr kommunikative und kooperative Zusammenarbeit, Förderung des Teamgeistes, Entstehen eines Teamgefühls in der Organisationseinheit vor Ort, Stärkung ,positiver“ Einstellungen. Diese Ergebnisse sprechen durchaus zu Gunsten interner Beratung als Qualifikation der Führungskräfte.

\section{Konsequenzen für internes Gruppen-Coaching}

Insgesamt lassen sich aus den Befunden einige Orientierungspunkte herausfiltern. Gruppen-Coaching im Bereich Führung und Management ist ein hochkomplexes, verdichtetes Personalentwicklungsprodukt. Es benötigt:

1. Eine Lernkultur des geschützten Rahmens (Regeln der Gruppenzusammenstellung, Diskretion, Verschwiegenheit bzgl. personenbezogener Informationen, Offenheit, Vertrauen),

2. Kompetenz in allen Fragen, die das Arbeits- und Berufsleben betreffen (umfassende Antwortkompetenz),

3. Kompetenz in feldspezifischen Managementfragen (Managementwissen im Fachbereich - von der Filiale bis zum Vorstand),

4. Psychologische Kompetenz (hochkompetente Coaching-Rolle),

5. Aktualität im Führungs- und Managementwissen (wissenschaftliche Ausbildung, ständig auf dem Laufenden sein in der wissenschaftlichen Diskussion), 
6. Pädagogische, gruppendynamische und Methodenkompetenz (an unterschiedliche Niveaus von Gruppen und komprimierte Zeit angepasste Trainings- und Inputeinheiten).

Der Zusammenhang scheint entscheidend zu sein. Ist eines der sechs Elemente nicht gewährleistet, wird kein Erfolg eintreten, weil aufgrund der Individualität der Wirkbedingungen alles bedient werden muss. Es scheint sich auch hier um notwendige Bedingungen zu handeln, die alle erfüllt sein müssen. Dann werden die konkreten Problemsituationen gelöst. Es entsteht eine dauerhaft interessante Veranstaltung. Die Bindung zur Maßnahme wird erhalten.

Zentraler Bezugspunkt des Coachings in der Gruppe ist außerdem die Ankoppelung an die Praxis des Führens und Managens. Der Alltag des Führens bietet laufend Anlässe und Möglichkeiten, die Führung reflektieren und verbessern lassen können. Die praxisnahen Lernprozesse leben von bestimmten Voraussetzungen. Zentral ist der geschützte Rahmen. Die Teilnehmer einer Coaching-Gruppe zu Führung lernen nicht primär eine Technik, sondern ihre eigene Person und Persönlichkeit in der Führungsbeziehung im Unternehmen einzusetzen. Reines Techniklernen bringt erfahrungsgemäß nichts, sonst wären studierte Betriebswirte per se gute Führungskräfte, denn sie müssen sich im Studium mit Führungskonzepten befassen. Führung lernen ist Persönlichkeitsentwicklung. Das aber braucht den geschützten, diskreten Rahmen. Diesen kann vom Prinzip her nur ein dazu berechtigter und autorisierter Leiter oder Coach herstellen, der dabei nicht mit anderen Rollen in Konflikt kommt. So ist z. B. ein Diplom-Psychologe von seinem Berufstand her zur Diskretion verpflichtet. Er kann die nötige Diskretion auch der Firma gegenüber gewährleisten. Alle in Führungspositionen oder Personalfunktionen mit disziplinarischen Entscheidungen betrauten Rollenträger können dies explizit nicht.

Ein zweiter wesentlicher Aspekt ist die professionelle Vielfalt der Arbeit in den Coaching-Gruppen. Orientiert am Professionalisierungsziel für eine Führungskraft, dem Quadrat aus Feldkompetenz, Methodenkompetenz, Theoriekompetenz und Personkompetenz (Erpenbeck und Rosenstiel 2007; Mohr 2008) erfährt eine Führungskraft in einer gut gestalteten, im Intervallprinzip aufgebauten Maßnahme ein integriertes Lernen. Dies setzt auf Seiten des Leiters der Gruppen ein umfassendes Beherrschen der genannten Professionalisierungsperspektiven voraus. Entsprechend hoch sind die Anforderungsbedingungen an den Leiter einer solchen Maßnahme. Dies umfasst Coaching- und Supervisions-Kompetenz, pädagogische Kompetenz, inhaltliches Auf-dem-Laufenden-Sein in moderner Führungstheorie und -forschung sowie Feldkompetenz zu den Arbeitsfeldern der Teilnehmer.

Dritter Aspekt ist die Coaching-Kompetenz des Leiters. Er muss in der Lage sein, Menschen in professionellen Umfeldern zu begleiten und ihr Wachstum zu unterstützen. Dies besteht v. a. auch in der Möglichkeit, in der knapp bemessenen Zeit der Coaching-Gruppe Freiräume und Gelegenheiten zur Erweiterung des eigenen Spektrums zu gewährleisten.

Nicht zu vernachlässigen ist auch das unternehmenskulturspezifische Wissen des Leiters. Es bezieht sich auf das Geschäft des Unternehmens in möglichst vielen seiner Dimensionen sowie auf das spezielle Unternehmen. Dies ermöglicht in der komprimier- 
ten Zeit passende Lösungen. Durch seine eigene Supervision sorgt der Leiter für die nötige innere Distanz, um nicht betriebsblind zu werden.

Open Access: Dieser Artikel unterliegt den Bedingungen der Creative Commons Attribution License. Dadurch sind die Nutzung, Verteilung und Reproduktion erlaubt, sofern der/die Originalautor/en und die Quelle angegeben sind.

\section{Literatur}

Bentrop, C., \& Schneider, M. (2012). Neo-Tayloristic or Holistic Work Group: Flexible Production and the Not So High Performance Work Practices in German Maschinenbau. Studie präsentiert bei der QCA-Konferenz der Universität Hamburg, 1. Juni 2012.

Berelson, B. (1952). Content analysis in communication research. Glencoe III: Free Press.

Berne, E. (1979). Struktur und Dynamik von Organisationen und Gruppen. München: Kindler.

Buer, F., \& Schmidt-Lellek, C. (2008). Life-Coaching. Über Sinn, Glück und Verantwortung in der Arbeit. Göttingen: Vandenhoeck \& Ruprecht.

Deutscher Bundesverband Coaching. (2010). Leitlinien und Empfehlungen für die Entwicklung von Coaching als Profession, Kompendium mit den Professionsstandards des DBVC (3., erw. Aufl.). Osnabrück: DBVC Geschäftsstelle.

Ennen, E., \& Richter, A. (2010). The whole is more than the sum of its parts - Or is it? A review of the empirical literature on complementaries in organizations. Journal of Management, 36(1), 207-233.

Erpenbeck, J., \& von Rosenstiel, L. (Hrsg.). (2007). Handbuch Kompetenzmessung. Stuttgart: Schäffer Poeschel.

Fiss, P. (2007). A set-theoretical approach to organizational configurations. Academy of Management Review, 32(4), 1180-1198.

Grawe, K. (1994). Psychotherapie ohne Grenzen. Verhaltenstherapie und psychosoziale Praxis, 26(3), 357-370.

Hagehülsmann, U., \& Hagehülsmann, H. (2001). Der Mensch im Spannungsfeld seiner Organisation. Paderborn: Junfermann.

Heinloth, S. (2011). Praxishandbuch für Führungskräfte. München: Hanser.

Hewitt, G. (1995). Cycles of psychotherapy. Transactional Analysis Journal, 25(3), 200-207.

Jackson, G. (2005). Employee representation in the board compared: A fuzzy sets analysis of corporate governance, unionism, and political institutions. Industrielle Beziehungen, 12(3), 1-28.

Katz, D., \& Kahn, R. L. (1978). The social psychology of organizations. Wiley.

Mayring, P. (2010). Qualitative Inhaltsanalyse (11., akt. u. überarb. Aufl.). Weinheim: Beltz.

Mohr, G. (2000). Lebendige Unternehmen führen. Frankfurt: FAZ-Buchverlag.

Mohr, G. (2006). Systemische Organisationsanalyse, Grundlagen und Dynamiken der Organisationsentwicklung. Bergisch Gladbach: EHP.

Mohr, G. (2007). Interne Beratung im Unternehmen. In H. Hagehülsmann (Hrsg.), Beratung zu professionellem Wachstum (S. 179-204). Paderborn: Junfermann.

Mohr, G. (2008). Coaching und Selbstcoaching mit Transaktionsanalyse. Professionelle Beratung zu beruflicher und persönlicher Entwicklung. Bergisch Gladbach: EHP.

Mohr, G. (2009). Wirtschaftskrise und neue Orientierung. Berlin: ProBusiness.

Mohr, G. (2010). Workbook Coaching und Organisationsentwicklung. Bergisch Gladbach: EHP.

Mohr, G. (2012). Ethik in einer Wirtschaft am seidenen Faden. Zeitschrift für Transaktionsanalyse, 29(2), 77-99. 
Ragin, C. (1987). The comparative method: Moving beyond qualitative and quantitative strategies. Berkeley: University of California Press.

Ragin, C. (2000). Fuzzy set social science. Chicago: University of Chicago Press.

Ragin, C. (2008). Redesigning social inquiry: Fuzzy sets and beyond. Chicago: University of Chicago Press.

Rauen, C. (2007). http://www.rauen.de/coaching-artikel/coaching-index-qualitaetsmodell.htm. Zugegriffen: 24. Mai. 2012.

Schmid, B. (2003). Systemische Professionalität und Transaktionsanalyse. Bergisch Gladbach: EHP.

Schmid, B., \& Messmer, A. (2005). Systemische Personal-, Organisations- und Kulturentwicklung. Bergisch Gladbach: EHP.

Schmidt, G. (2005). Einführung in die hypnosystemische Therapie und Beratung. Heidelberg: Auer.

Schmidt-Lellek, C., \& Buer, F. (Hrsg.). (2011) Life-Coaching in der Praxis. Wie Coaches umfassend beraten. Göttingen: Vandenhoek \& Ruprecht.

Shazer de, S. (1990). Der Dreh. Überraschende Wendungen und Lösungen in der Kurzzeittherapie. Heidelberg: Auer.

Wagemann, C., \& Schneider, C. (2007). Qualitative Comparative Analysis (QCA) und Fuzzy Sets: Ein Lehrbuch für Anwender und jene, die es werden wollen. Opladen: Budrich.

Zur Bonsen, M. (2009). Leading with life: Lebendigkeit im Unternehmen freisetzen und nutzen. Wiesbaden: Gabler.

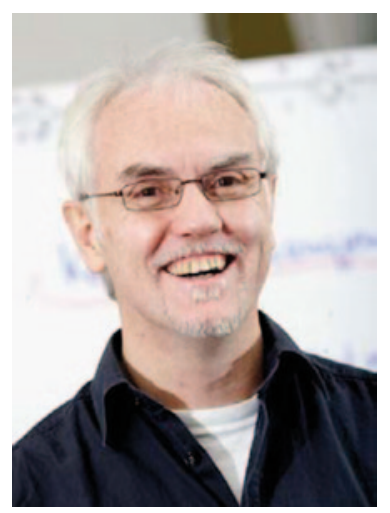

Günther Mohr, Diplom-Psychologe, Diplom-Volkswirt, Senior Coach DBVC, Supervisor BDP, Lehrender Transaktionsanalytiker (ITAA, DGTA), Institut für Coaching, Training und Consulting, Hofheim b. Frankfurt, www.mohr-coaching.de. 